\title{
PAPER
}

\section{Fiber Bragg grating hydrophone with polarization-maintaining fiber for mitigation of polarization-induced fading}

\author{
Kenji Saijyou $^{1, *}$, Chiaki Okawara ${ }^{2}$, Tomonao Okuyama ${ }^{3}$, \\ Yasuyuki Nakajima ${ }^{4}$ and Ryotaku Sato ${ }^{4}$ \\ ${ }^{1}$ Department of Naval Systems Development, Technical R\&D Institute, Ministry of Defense, \\ 5-1 Ichigaya-Honmuracho, Shinjuku-ku, Tokyo, 162-8830 Japan \\ ${ }^{2}$ Defense Policy Division, Bureau of Defense Policy, Ministry of Defense, \\ 5-1 Ichigaya-Honmuracho, Shinjuku-ku, Tokyo, 162-8830 Japan \\ ${ }^{3}$ Naval Systems Research Center, Technical R\&D Institute, Ministry of Defense, \\ 3-13-1 Nagase, Yokosuka, 239-0826 Japan \\ ${ }^{4}$ Defense Systems Div., Oki Electric Industry Co., Ltd., \\ 688 Ozuwa Numazu, 410-0873 Japan
}

(Received 1 August 2011, Accepted for publication 4 January 2012)

\begin{abstract}
In this paper, we describe an interferometric optical fiber hydrophone using a pair of fiber Bragg gratings (FBGs) with a polarization-maintaining fiber (PMF). Signal fading induced by random fluctuations in the state of polarization for interfering beams of a fiber interferometer is a common problem for all interferometric optical fiber sensors. To overcome this signal fading problem, a PMF was utilized to construct an interferometric optical fiber hydrophone using a pair of FBGs. Then the performance of the PMF hydrophone was compared with that of a conventional single-mode optical fiber hydrophone. In our experiment, we adopted a $3 \times 3$ coupler scheme for the demodulation of acoustic signals to reduce the computational load required for the demodulation scheme, and the effectiveness of the proposed optical fiber hydrophone with the $3 \times 3$ coupler scheme was confirmed.
\end{abstract}

Keywords: Optical fiber hydrophone, Fiber Bragg grating, Polarization maintaining fiber, Signal fading induced by polarization

PACS number: 43.38.Ar, 43.30.Yj, 43.38.Zp, 43.30.Xm [doi:10.1250/ast.33.239]

\section{INTRODUCTION}

Interferometric optical fiber technologies have been studied by many researchers because they have a number of advantages over conventional electrical sensing technologies, making them attractive for use over a wide range of application areas. In 1990, a Fabry-Perot interferometer using pairs of fiber Bragg gratings (FBGs) as reflectors was proposed [1]. The major application of interferometric optical fiber technologies is to optical-fiber hydrophones $[2,3]$. To develop techniques that allow multiple-hydrophone arrays, time division multiplexing (TDM) [4] and wavelength division multiplexing (WDM) [5-7] were proposed, and large-scale TDM and WDM schemes for interferometric optical fiber hydrophones were demonstrated $[1,8]$.

\footnotetext{
*e-mail: saijyou-kenji@jcom.home.ne.jp
}

Okawara and Saijyou proposed an interferometric optical fiber hydrophone with a pairs of FBGs as reflectors (abbreviated as "FBG-based hydrophone," hereafter) in their papers $[9,10]$. The use of FBGs as partial reflectors and wavelength selectors in interferometric optical fiber hydrophones simplifies the structure of the hydrophone array to reduce the numbers of couplers, joints, and other interconnects required in other types of optical fiber hydrophones for constructing an array.

In order to realize a large-scale array of FBG-based hydrophones, there are some problems which must be solved: (1) how to multiplex acoustic signals, (2) how to reduce the influence of polarization-induced fading (PIF) $[11,12]$, (3) the type of demodulation scheme that is appropriate for them.

To solve the first problem, the TDM and WDM schemes were adopted by Okawara and Saijyou and they revealed the effectiveness of these schemes $[9,10]$. 


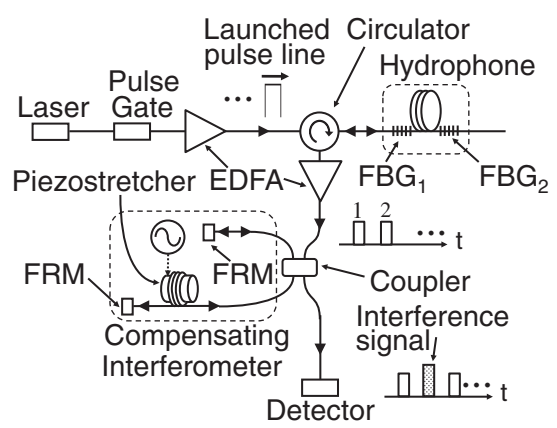

Fig. 1 Configuration of the FBG-based hydrophone. The demodulation scheme is the PGC scheme.

In this paper, a method of reducing the influence of the PIF and a demodulation scheme are discussed. In the next section, we propose to adopt of the $3 \times 3$ coupler scheme [13] for the demodulation of acoustic signals, and compare it with the phase-generated carrier (PGC) scheme [14-16]. Furthermore, methods of mitigating the PIF are explained in section 3 .

\section{DEMODULATION SCHEMES OF FBG-BASED HYDROPHONE}

\subsection{Phase-Generated Carrier Scheme with a Path- Matched Differential Interferometry}

Figure 1 shows the basic configuration of a conventional FBG-based hydrophone system [8]. At the detector, the intensity of the interference signal is given by

$$
\begin{aligned}
I= & I_{0}\left[1+V \cos \left(A \cos \omega_{\mathrm{PGC}} t+\phi(t)\right)\right] \\
= & I_{0}\left\{1+\left[J_{0}(C)+2 \sum_{k=1}^{\infty}(-1)^{k} J_{2 k}(C)\right.\right. \\
& \left.\times \cos 2 k \omega_{\mathrm{PGC}} t\right] \cos \phi(t) \\
& -\left[2 \sum_{k=0}^{\infty}(-1)^{k} J_{2 k+1}(C)\right. \\
& \left.\left.\times \cos (2 k+1) \omega_{\mathrm{PGC}} t\right] \sin \phi(t)\right\}
\end{aligned}
$$

where $I_{0}$ is the average intensity of the interference signal, $V$ is the visibility $(0 \leq V \leq 1), \omega_{\mathrm{PGC}}$ and $A$ are the frequency and the amplitude of sinusoidal modulation imposed on the compensating interferometer by a piezostretcher, and $\phi(t)$ is the acoustic signal for demodulation, respectively. This demodulation scheme is called the "phase-generated carrier (PGC) scheme with path-matched differential interferometry (PMDI)" (abbreviated as the "PGC scheme," hereafter), and is widely applied to optical fiber interferometric sensor systems [2,14-16].

To apply the PGC scheme, the condition of $\omega_{\mathrm{PGC}} \geq$ $8 \omega_{0}$ must be satisfied, where $\omega_{0}$ is the highest frequency

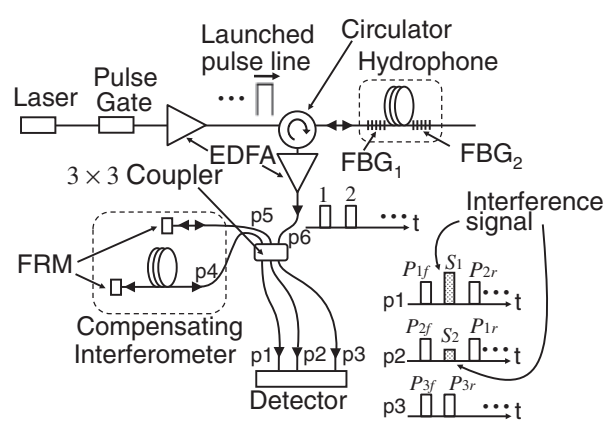

Fig. 2 Configuration of the $3 \times 3$ coupler scheme.

of the acoustic signal, and the two terms, $\cos \omega_{\mathrm{PGC}} t$ and $\cos 2 \omega_{\mathrm{PGC}} t$, are required for the demodulation process of the PGC scheme. Therefore, relatively large computational capability is required for the PGC scheme. This requirement becomes a disadvantage of the PGC scheme.

\subsection{Configuration of $3 \times 3$ Coupler Scheme with PMDI}

Figure 2 shows the basic configuration of the $3 \times 3$ coupler scheme with the PMDI (abbreviated as the " $3 \times 3$ coupler scheme," hereafter). The two outputs of interference signals of ports 1 and 2 of the $3 \times 3$ coupler are given by

$$
\begin{aligned}
& S_{1}=A_{1}\left[1+V_{1} \cos (\phi(t)+\pi / 3)\right], \\
& S_{2}=A_{2}\left[1+V_{2} \cos (\phi(t)-\pi / 3)\right],
\end{aligned}
$$

where $A_{1}$ and $A_{2}$ are average intensities of interference signals, and $V_{1}$ and $V_{2}$ are their visibilities $\left(0 \leq V_{1}\right.$, $V_{2} \leq 1$ ), respectively. Orthogonal components of the acoustic signal are derived from the interference signals $S_{1}$ and $S_{2}$ as

$$
\begin{aligned}
\cos \phi(t) & =\frac{S_{1}-A_{1}}{A_{1} V_{1}}+\frac{S_{2}-A_{2}}{A_{2} V_{2}}, \\
\sin \phi(t) & =\frac{1}{\sqrt{3}}\left[\frac{S_{2}-A_{2}}{A_{2} V_{2}}-\frac{S_{1}-A_{1}}{A_{1} V_{1}}\right] .
\end{aligned}
$$

Therefore, $\phi(t)$ can be obtained from

$$
\phi(t)=\tan ^{-1} \frac{\sin \phi(t)}{\cos \phi(t)} .
$$

This is the basic concept of the $3 \times 3$ coupler scheme [13]. In this scheme, there are no active components in the compensating interferometer. Also, the sampling frequency of the interference signals can be selected to be far lower than that of the PGC scheme. These are advantages of the $3 \times 3$ coupler scheme.

To apply this demodulation scheme, the average intensities of interference signals and the visibilities should be obtained. The derivation of these variables is described as follows. The interference signal of port $1\left(S_{1}\right)$ can be rewritten as 


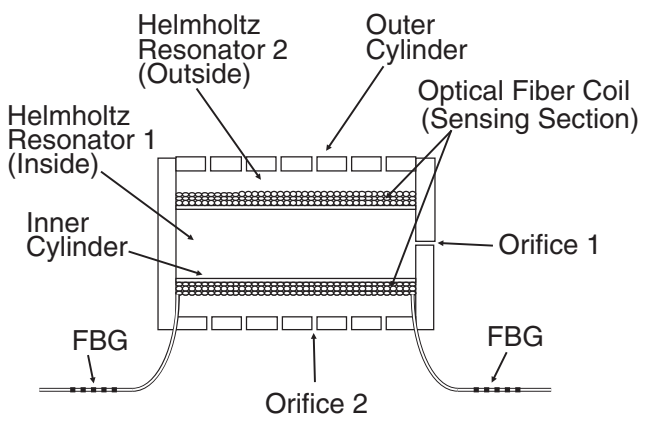

Fig. 3 Internal structure of the FBG-based hydrophone.

$$
\begin{aligned}
S_{1} & =A_{1}\left[1+V_{1} \cos (\phi(t)+\pi / 3)\right], \\
& =c_{1}\left[\sqrt{r_{1} l_{2}} \exp (i \omega t)+\sqrt{s_{2} l_{1}} \exp (i \omega t+\phi(t)+\pi / 3)\right]^{2}, \\
& =c_{1}\left[r_{1} l_{2}+s_{2} l_{1}+2 \sqrt{r_{1} l_{1} s_{2} l_{2}} \cos (\phi(t)+\pi / 3)\right],
\end{aligned}
$$

where $r_{1}$ and $r_{2}$ are reflectance factors of $\mathrm{FBG}_{1}$ and $\mathrm{FBG}_{2}$, $l_{1}$ can be written as $l_{1}=\left(1-r_{1}\right)^{2} l_{1}^{2} r_{2}, l_{1}^{\prime}$ is the transmission loss between $\mathrm{FBG}_{1}$ and $\mathrm{FBG}_{2}, s_{2}$ and $l_{2}$ are internal losses for the short and long arms of the compensating interferometer, and $c_{n}$ is the insertion loss of the $3 \times 3$ coupler, in which subindex $n$ indicates the output port of the $3 \times 3$ coupler, respectively. Here, the intensities of output pulses shown in Fig. 2 are given by

$$
\begin{aligned}
& P_{1 f}=c_{1} r_{1} s_{2}, \quad P_{1 r}=c_{1} l_{1} l_{2}, \\
& P_{3 f}=c_{3} r_{1}, \quad P_{3 r}=c_{3} l_{1} .
\end{aligned}
$$

From them, $A_{1}$ and $V_{1}$ can be obtained as

$$
\begin{aligned}
& A_{1}=c_{1}\left(r_{1} l_{2}+s_{2} l_{1}\right)=\frac{P_{1 f} P_{3 r}}{P_{3 f}}+\frac{P_{1 r} P_{3 f}}{P_{3 r}}=\frac{P_{1 f}}{R}+P_{1 r} R, \\
& V_{1}=2 c_{1} \frac{\sqrt{r_{1} l_{1} s_{2} l_{2}}}{A_{1}}=\frac{2 \sqrt{P_{1 f} P_{1 r}}}{A_{1}}, \quad R=\frac{P_{3 f}}{P_{3 r}} .
\end{aligned}
$$

Similarly, $A_{2}$ and $V_{2}$ become

$$
A_{2}=\frac{P_{2 f}}{R}+P_{2 r} R, \quad V_{2}=\frac{2 \sqrt{P_{2 f} P_{2 r}}}{A_{2}} .
$$

\section{MITIGATION METHODS OF SIGNAL FADING INDUCED BY POLARIZATION}

At first, we describe the mechanism behind of PIF. Figure 3 shows the internal structure of the FBG-based hydrophone, in which Helmholtz resonators are constructed by the inside and outside sensing fibers wound around the hydrophone on the cylinder. The dynamic cylinder radial strain, which is induced by the difference in pressure between the inside and outside cylinders, yields fiber optic path length variations determined using PGC or $3 \times 3$ coupler schemes. An asymmetrical side pressure is added to the optical fiber between FBGs, thus the state of polarizations (SOPs) of returned pulses are varied when the

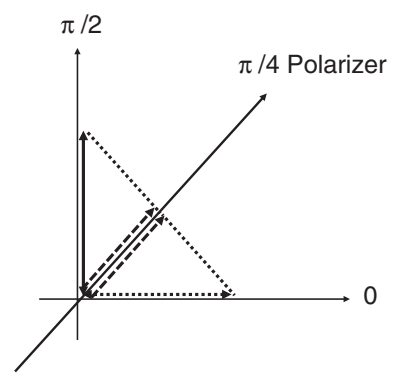

Fig. 4 Technique of using polarization diversity receiver. $\pi / 4$ polarizer is applied. A couple of pulses, which are orthogonal to each other, can interfere with each other through the $\pi / 4$ polarizer.

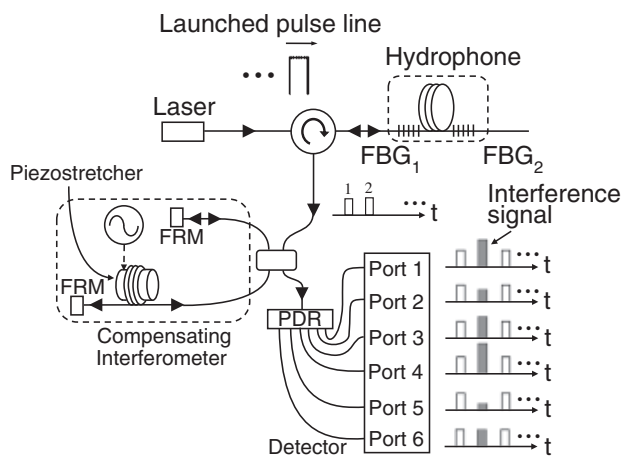

Fig. 5 Configuration of the FBG-based hydrophone system with 6-port PDR. Acoustic signals are demodulated by the PGC scheme.

fiber optical path length is changed. If SOPs of recombined pulses are identical, they will induce optimal interference and the visibilities will become unity. However if they are orthogonal, there is no interference between them, and thus the visibilities become zero. This phenomenon is PIF, which causes an increase in the sensor noise.

To overcome this PIF phenomenon, a technique of using a polarization diversity receiver (PDR) is utilized. Figure 4 shows the basic concept of the technique of using the PDR (called as the "PDR method," hereafter) [2,17]. If the SOP of one pulse is $0 \mathrm{rad}$ (dotted line in Fig. 4) and the other is $\pi / 2 \mathrm{rad}$ (solid line in Fig. 4), the visibilities of interference signals become zero without using a PDR. However, a $\pi / 4$ polarizer produces a signal since both SOPs have a projection along the $\pi / 4$ axis.

Figure 5 shows the configuration of the FBG-based hydrophone system with a 6-port PDR. In this system, the PGC scheme is utilized for the demodulation of acoustic signals. To reduce the influence of the PIF, the output of the PDR port with the maximum visibility is demodulated. When environment parameters (temperature and/or pressure) of the hydrophone vary, the selected port is changed because of the fluctuation of the SOPs. If the variations in environment parameters for the hydrophone are small, then 


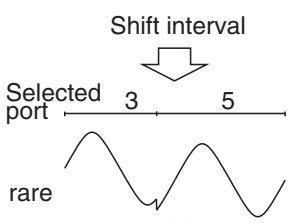

(a)

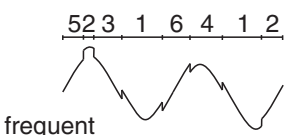

(b)

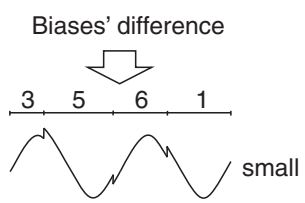

(c)

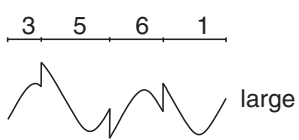

(d)
Fig. 6 Typical influences of the shift interval and the phase bias difference on the demodulated signal. (a) Shift interval is rare. (b) Shift interval is frequent. (c) Phase bias difference is small. (d) Phase bias difference is large.

the shift interval of the selected port becomes long, and vice versa.

Assuming that the phase of a demodulated signal does not depend on the selected port, the influence of the shift interval of the selected port can be neglected. However, if the phase of the demodulated signal does depend on the selected port, then a phase bias difference exists between each port of the PDR, and this phase bias difference induces noise in the demodulated signal. The mechanism of generating noise (called "noise induced by the phase bias difference," hereafter) can be explained as follows.

Figure 6 illustrates the influence of the shift interval and the phase bias difference. Figures 6(a) and 6(b) show the influence of the shift interval with phase bias difference being constant. When the shift interval of the selected port is long (Fig. 6(a)), discrepancies between the demodulated signals are rare, and the noise level induced by the change of the selected port becomes low. On the other hand, when the shift interval of the selected port is short (Fig. 6(b)), the noise level becomes high.

Figures 6(c) and 6(d) show the cases when the shift interval is constant. In this case, when the phase bias difference is small (Fig. 6(c)), discrepancies between the demodulated signal become small, leading to a low noise level of the demodulated signal. On the other hand, when the phase bias difference is large (Fig. 6(d)), the noise level becomes high. The noise level induced by the phase bias difference is summarized as

$$
\text { Noise }_{\mathrm{PBD}}(f)=20 \log _{10}\left(\frac{a}{\pi f T}\right)-R S(f),
$$

where $a[\mathrm{rad}]$ is the average phase bias difference, $T$ [s] is the average shift interval of the selected port, and $R S(f)$ is the receiving sensitivity (RS). The derivation process of Eq. (9) is described in Appendix. To overcome this difficulty, the polarization-maintaining fiber (PMF) is

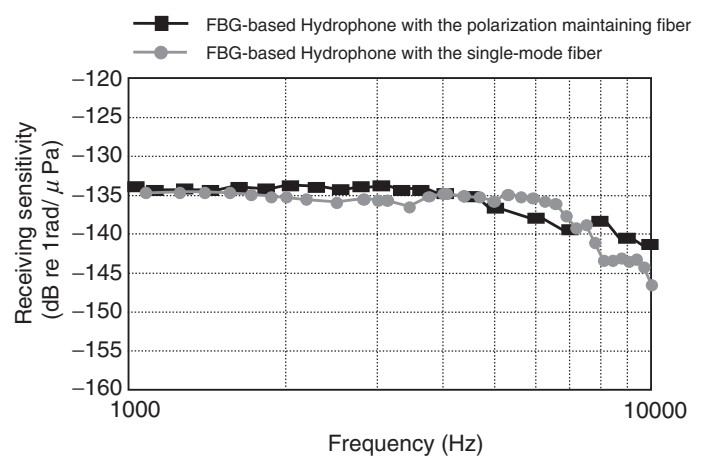

Fig. 7 Receiving sensitivity of the PMF hydrophone and that of the SMF hydrophone.

utilized to make the optical-fiber hydrophone system. As the SOP is not varied in the PMF, the influence of PIF is negligibly small. Furthermore, a multiport PDR is not necessary, i.e., the noise explained above does not exist.

\section{EXPERIMENTAL RESULTS}

\subsection{Experimental Apparatus}

To demonstrate the effectiveness of the FBG-based hydrophone with the PMF (abbreviated as "PMF hydrophone," hereafter) and the $3 \times 3$ coupler scheme, a prototype of the hydrophone was manufactured and its performance was compared with that of the FBG-based hydrophone with a single-mode fiber (SMF) (abbreviated as "SMF hydrophone," hereafter). The experiment was conducted at the Naval Systems Research Center of the Ministry of Defense's water pool, which is $15 \mathrm{~m}$ long, $9 \mathrm{~m}$ wide, and $8 \mathrm{~m}$ deep. The laser wavelength was $1551.72 \mathrm{~nm}$, the optical power level was $+20 \mathrm{dBm}$, and the optical fiber length between FBGs of the hydrophone was $130 \mathrm{~m}$. The AdjustiK-E15 (NKT Photonics) laser, IPSAD-1502 (Inphenix) pulse gate, AEDFA-PM-23 (Amonics) EDFA, and TIA-525i (TTi) detector were used.

\subsection{Receiving Sensitivity of Hydrophone}

First, the validity of the $3 \times 3$ coupler scheme for the FBG-based hydrophone was examined. Figure 7 shows RS of the prototype of the PMF hydrophone and that of the SMF hydrophone. As the environmental temperature was $15^{\circ} \mathrm{C}$, the variation of the temperature was negligible, therefore it was assumed that the environmental conditions were in a steady state. An acoustic signal of the PMF hydrophone was demodulated by the $3 \times 3$ coupler scheme, however, that of the SMF hydrophone was demodulated by the conventional PGC scheme. We set the frequency range (the deviation of RS was less than $3 \mathrm{~dB}$ ) for both hydrophones to be from 100 to $5,000 \mathrm{~Hz}$, and the RS to be $-135 \mathrm{~dB}$ re $1 \mathrm{rad} / \mu \mathrm{Pa}$. Because of the limitation of the test basin size, the RS was measured from 1 to $10 \mathrm{kHz}$. The difference between measured RS's of the PMF hydrophone 


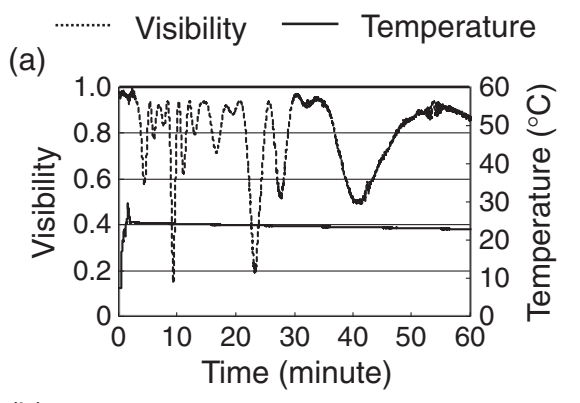

(b)

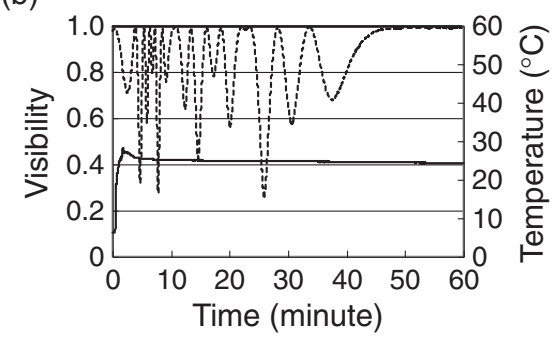

(c)

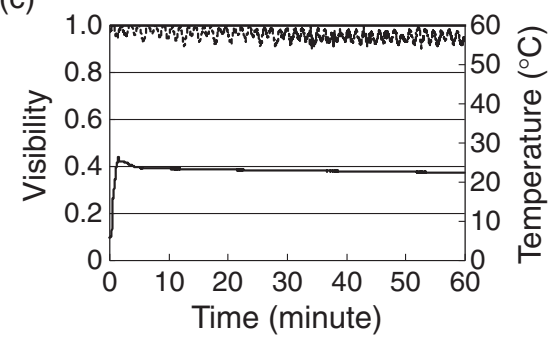

(d)

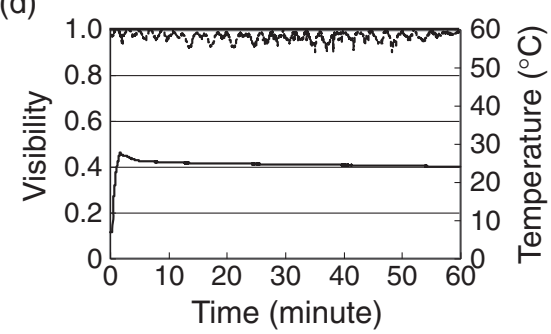

Fig. 8 Relationships between the visibility and the temperature surrounding the FBG-based hydrophone. (a) SMF hydrophone $(3 \times 3$ coupler scheme). (b) SMF hydrophone (PGC scheme). (c) PMF hydrophone $(3 \times 3$ coupler scheme). (d) PMF hydrophone (PGC scheme).

and the SMF hydrophone was negligible. This result confirmed the validity of the $3 \times 3$ coupler scheme for the FBG-based hydrophone.

\subsection{Relationship between Visibility and Variation of Environmental Parameters}

Next, the relationships between the visibility and the variation of environment parameters for these hydrophones were examined. Figure 8 shows the influence of the temperature variation on the visibility. The measurement system configuration for obtaining the data in Figs. 8(a) and 8(c) was based on Fig. 2, and that for obtaining the data in Figs. 8(b) and 8(d) was based on Fig. 5. We selected port 1 for all visibility measurements.
Figures 8(a) and 8(b) show the visibility of the SMF hydrophone. The demodulation scheme for obtaining the data shown in Fig. 8 (a) was the $3 \times 3$ coupler scheme and that in the case of Fig. 8(b) was the PGC scheme. First, we set the environment temperature to change rapidly from 5 to $30^{\circ} \mathrm{C}$ within one minute at the beginning of the measurement. Then, we maintained the environment at a relatively constant temperature. The visibility of the SMF hydrophone was varied, but the lowest visibility was less than 0.2. It can be shown that the influence of the temperature variation on the visibility of the SMF hydrophone lasts even after the temperature becomes constant. As the signal-to-noise ratio of the demodulated acoustic signal is inversely proportional to the visibility, the signalto-noise ratio of the demodulated acoustic signal can be reduced by more than $7 \mathrm{~dB}$ when the visibility becomes less than 0.2. This reduction of the visibility does not depend on the demodulated scheme.

Figures $8(\mathrm{c})$ and $8(\mathrm{~d})$ show the visibility of the PMF fiber hydrophone. The demodulation scheme for obtaining the data in Fig. 8(c) was the $3 \times 3$ coupler scheme and that in the case of Fig. 8(d) was the PGC scheme. The environment temperature varied as the same as that in the case of Figs. 8(a) and 8(b). The visibility of the PMF hydrophone was almost constant even though the environment temperature rapidly changed. The lowest visibility was 0.9 . This property does not depend on the demodulation scheme, where the fluctuation of visibility was about 0.05 , which was induced by the influence of the polarization extinction ratio (PER). This can be explained as follows.

As common PERs of optical devices are $20 \mathrm{~dB}, 99 \%$ of light power is maintained at the main polarization axis through these devices. There are five optical devices (laser source, pulse gate, two EDFAs, and circulator) in the configuration of the FBG-based hydrophone. In this case, the ratio of light power, which is maintained at the main polarization axis, can be decreased to $95 \%\left(0.99^{5} \approx 0.95\right)$. Therefore, the fluctuation of visibility becomes 0.05 .

Figure 9 shows the influence of pressure variation on visibility. The measurement configuration was the same as that in the case of Fig. 8. Figures 9(a) and 9(b) show the visibility of the SMF hydrophone. The demodulation scheme in the case of Fig. 9(a) was the $3 \times 3$ coupler scheme and that in the case of Fig. 9(b) was the PGC scheme. We changed the environment pressure rapidly within one minute at the beginning of the measurement, and subsequently, the environment pressure stayed almost constant. The visibility of the SMF hydrophone varied from 1 to 0.3 . These results show that the influence of pressure variation on the visibility of the SMF hydrophone continued even after the pressure became constant. This property did not depend on the demodulation scheme. 


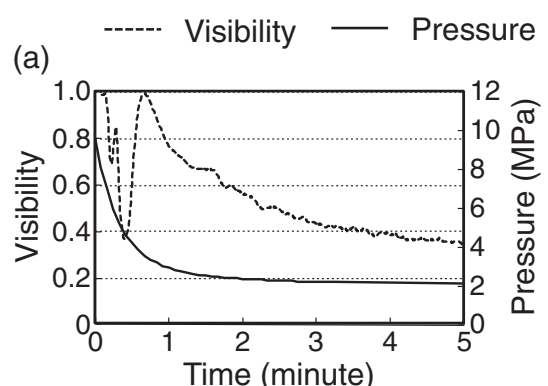

(b)

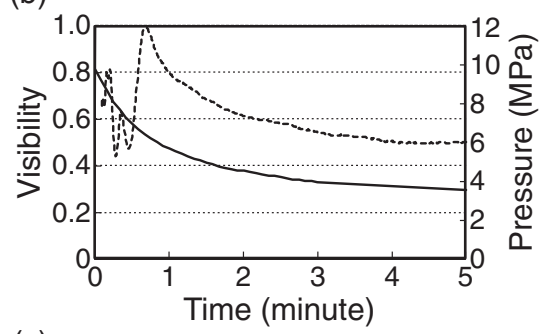

(c)
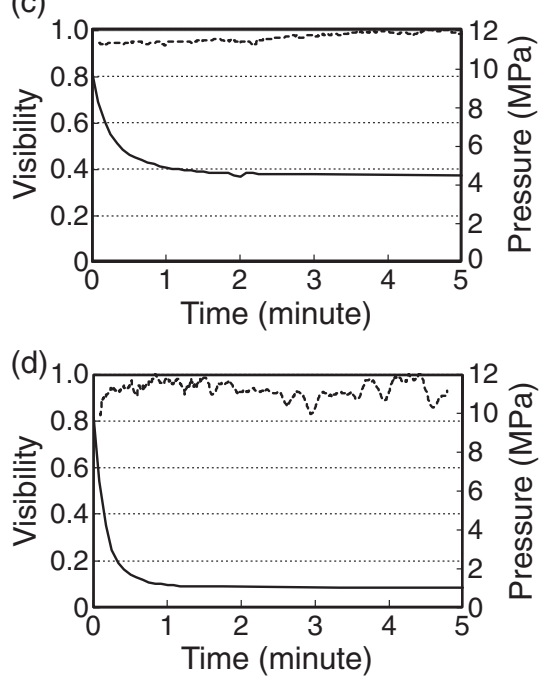

Fig. 9 Relationships between the visibility and the pressure surrounding the FBG-based hydrophone. (a) SMF hydrophone $(3 \times 3$ coupler scheme). (b) SMF hydrophone (PGC scheme). (c) PMF hydrophone $(3 \times 3$ coupler scheme). (d) PMF hydrophone (PGC scheme).

Figures 9(c) and 9(d) shows the visibility of the PMF hydrophone. The demodulation scheme in the case of Fig. 9(c) was the $3 \times 3$ coupler scheme and that in the case of Fig. 9(d) was the PGC scheme. The environment pressure change was the same as in the case of Figs. 9(a) and 9(b). The visibility of the PMF hydrophone stayed almost constant even though the environment pressure was rapidly changed, and the lowest visibility was 0.8 . This property did not depend on the demodulation scheme. These results show that the influence of the change in the hydrophone environment is strong for the SMF hydrophone, thus this influence is negligible for the PMF hydrophone.

The environment pressure change was different for Figs. 9(a)-9(d), however, the change of environment pressure directly propagated inside the hydrophone, the (a)

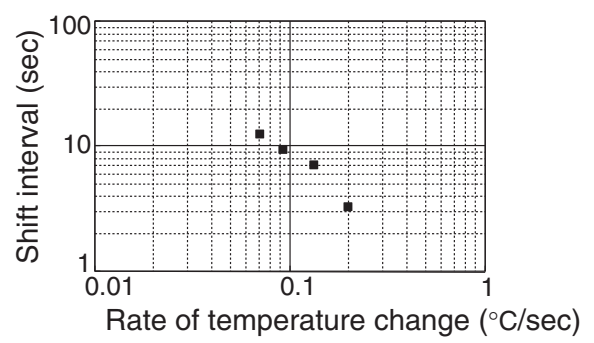

(b)

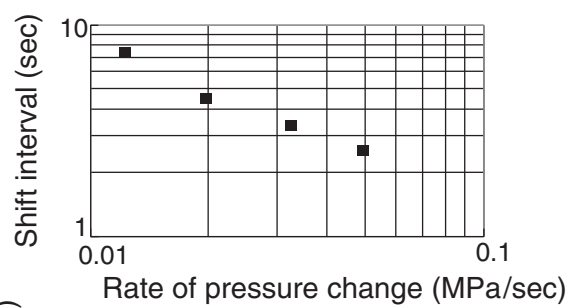

(c)

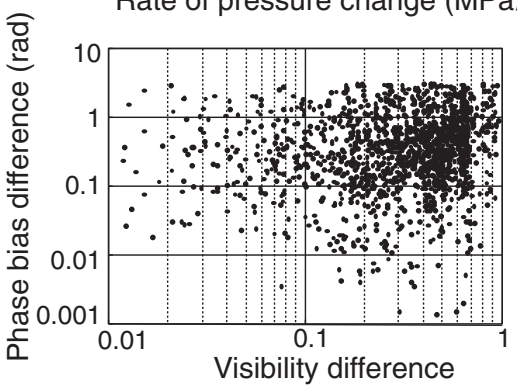

Fig. 10 Phase differences of the demodulated signals and the shift interval of the selected port. (a) Shift interval versus the rate of temperature change. (b) Shift interval versus the rate of pressure change. (c) Phase bias difference versus the visibility difference.

same as in the case of the acoustic signal. Therefore, the influence of the difference in environment pressure change was negligible.

\subsection{Demodulation Noise}

Next, we examined the noise induced by the PDR method with different phase bias. Figure 10 shows the shift interval of the selected port and the phase bias difference of the demodulated signals. The measurement configuration was the same as that in Fig. 5, where the hydrophone is the SMF hydrophone. Figure 10(a) illustrates the relationship between the shift interval of the selected port and the rate of change of temperature. The shift interval was inversely proportional to the rate of change of temperature. Figure 10(b) shows the relationship between the shift interval of the selected port and the rate of pressure change. The shift interval was in inverse proportion to the rate of pressure change. Figure 10(c) shows the relationship between the phase bias difference of demodulated signals and the visibility difference between the two port. It is considered that there is no correlation between the phase bias difference and the visibility difference. The average phase bias difference was $0.35 \mathrm{rad}$. From this result, the noise, induced in the PDR method of the SMF 


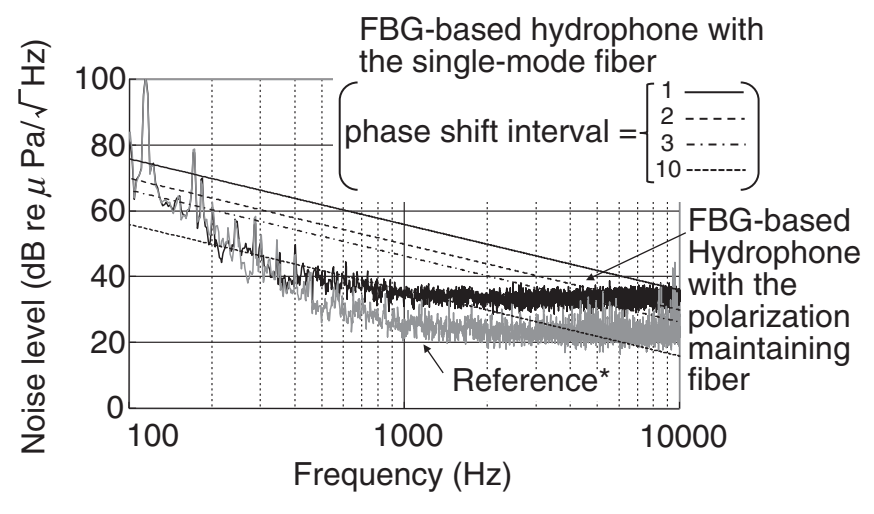

Fig. 11 Demodulation noise of FBG-based hydrophone. The reference is the conventional hydrophone (PP-143 made by Oki Electric Industry Co. Ltd.).

hydrophone with a phase bias difference, can be estimated as

$$
\begin{aligned}
\text { Noise }_{\mathrm{PBD}}(f)= & 20 \log _{10}\left(\frac{0.35}{\pi f T}\right) \\
& +135(\mathrm{~dB} \operatorname{re} 1 \mu \mathrm{Pa} / \sqrt{\mathrm{Hz}}) .
\end{aligned}
$$

Finally, the demodulation noise of the FBG-based hydrophone was examined. Figure 11 shows the noise of the FBG-based hydrophone. The black line shows the measured noise of the PMF hydrophone, which was demodulated by the $3 \times 3$ coupler scheme. The reference hydrophone was a conventional hydrophone, which measured the ambient noise in the test basin. The measured noise level of the PMF hydrophone was sufficiently low, and it was the same level as that of the SMF hydrophone in the steady-state environment [10]. Hence, the PMF hydrophone and the SMF hydrophone can be utilized to make sonar systems for a steady-state environment.

However, if the environment is not in a steady state, the noise level of the SMF hydrophone becomes high. Straight lines in Fig. 11 represent estimated demodulation noise of the SMF hydrophone. The estimated noise induced in the PDR method with the phase bias difference was obtained using Eq. (10). If the shift interval of the selected port is $10 \mathrm{~s}$, the noise induced by the phase bias difference becomes negligible. However, if the shift interval is $3 \mathrm{~s}$, the noise level cannot be neglected. If the rate of change of temperature is $0.2^{\circ} \mathrm{C} / \mathrm{s}$, the shift interval becomes $3 \mathrm{~s}$. In contrast to this, the noise level of the PMF hydrophone does not become large even when the environment is not in a steady state. Thus, the PMF hydrophone is considered to be appropriate for making sonar systems in non steadystate environments.

\section{CONCLUSION}

In our research, we showed the performance of the interferometric optical fiber hydrophone based on FBG with a PMF. A serious problem in the development of all interferometric optical fiber sensors is PIF. Thus, using a PDR is a common technique of alleviating PIF. This method is considered to be appropriate for making a sonar system in the steady-state environment. However, if the environment around the hydrophone is perturbed, the phase bias difference between demodulated phases of the ports of the PDR generates noise within the demodulated signal.

To overcome this difficulty, we proposed the utilization of PMF to make the FBG-based hydrophone. Additionally, to reduce the required computational load for the demodulation process, the application of the $3 \times 3$ coupler scheme was proposed. The effectiveness of the proposed method was demonstrated by the following experiment results.

(1) The visibility of the FBG-based hydrophone with the SMF varied when the environment condition was perturbed. In contrast, the visibility of the FBG-based hydrophone with the PMF was almost constant even when the environment condition was perturbed.

(2) The noise level of the FBG-based hydrophone with the SMF could not be neglected in the non steadystate environment. In contrast, the noise level of the FBG-based hydrophone with the PMF was sufficiently low not only in the steady-state environment but also in the non steady-state environment.

From the above results, we confirmed the effectiveness of the proposed method and recommended its use in making a sonar system not only for a steady-state environment, but also for the non steady-state environment.

\section{REFERENCES}

[1] W. W. Morey, "Distributed fiber grating sensors," Proc. OFS '90, Sydney, Australia, pp. 285-288 (1990).

[2] G. A. Cranch, P. J. Nash and C. K. Kirkendall, "Large-scale remotely interrogated arrays of fiber-optic interferometric sensors for underwater acoustic applications," IEEE Sens. J., 3, 19-30 (2003).

[3] G. A. Cranch, R. Crickmore, C. K. Kindendall, A. Bautista, K. Daley, S. Motley, J. Salzano, J. Latchem and P. J. Nash, "Acoustic performance of a large-aperture, seabed, fiber-optic hydrophone array," J. Acoust. Soc. Am., 115, 2848-2858 (2004).

[4] B. Culshaw and J. Dakin, Optical Fiber Sensors: Systems and Applications, Vol. 2 (Artech House, 1989).

[5] K. Fussgaenger, W. Koester, H. D. Saller and T. Vollmer, " $4 \times 560 \mathrm{Mbit} / \mathrm{s}$ DWM system using 3 wavelength selective fused single-mode couplers as multiplexer," Proc. ECOC ' 86 (1986).

[6] H. Ishio, J. Minowa and K. Nosu, "Review and status of wavelength-division multiplexing technology and its application," J. Lightwave Technol., LT-2, 448-463 (1984).

[7] G. Winzer, "Wavelength multiplxing components - A review of single-mode devises and their applications," J. Lightwave Technol., LT-2, 369-378 (1984).

[8] G. A. Cranch and P. J. Nash, "Large-scale multiplexing of interferometric fiber-optic sensors using TDM and DWDM," J. Lightwave Technol., 19, 687-699 (2001).

[9] C. Okawara and K. Saijyou, "Fiber optic interferometric 
hydrophone using fiber Bragg grating with time division multiplexing," Acoust. Sci. \& Tech., 28, 39-42 (2007).

[10] C. Okawara and K. Saijyou, "Fiber optic interferometric hydrophone using fiber Bragg grating with wavelength division multiplexing," Acoust. Sci. \& Tech., 29, 232-234 (2007).

[11] S. K. Sheem and T. G. Giallorenzi, "Polarization effects on single mode optical fiber sensors," Appl. Phys. Lett., 35, 914917 (1979).

[12] D. W. Stowe, D. R. Moore and R. G. Priest, "Polarization fading in fiber interferometric sensors," J. Quantum Electron., QE-18, 1644-1647 (1982).

[13] Z. Zhao, M. S. Demokan and M. MacAlpine, "Improved demodulation scheme for fiber optic interferometers using an asymmetric $3 \times 3$ coupler," J. Lightwave Technol., 15, 2059 2068 (1997).

[14] A. Dandridge, A. B. Tveten and T. G. Giallorenzi, "Homodyne demodulation scheme for fiber optic sensors using phase generated carrier," IEEE J. Quantum Electron., 18, 1647-1653 (1982).

[15] S.-C. Huang, W.-W. Lin and M.-H. Chen, "Phase sensitivity normalization in time-division multiplexing of polarizationinsensitive interferometric sensors using phase-generated carrier demodulation," Opt. Eng., 35, 2634-2640 (1996).

[16] A. D. Kersey, A. Dandridge and A. B. Tveten, "Time-division multiplexing of interferometric fiber sensors using passive phase-generated carrier interrogation," Opt. Lett., 12, 775-777 (1987).

[17] N. J. Frigo, A. Dandridge and A. B. Tveten, "Technique for elimination of polarization fading in fiber interferometers," Electron. Lett., 20, 319-320 (1984).

\section{Appendix: DERIVATION OF NOISE LEVEL INDUCED BY PHASE BIAS DIFFERENCE}

When a selected port of the PDR is changed, noise induced by the phase bias difference is added to the demodulated signal. Therefore, if the selected port is changed once every period of a fast Fourier transform (FFT), the noise spectrum of the phase bias difference of the demodulated signal can be represented as the Fourier transformation of the Heaviside step function. When the change of the selected port occurs at $t=0$, the phase bias difference of the demodulated signal is described as

$$
\text { noise }_{\text {def }}(t)=a \times h(t)=\left\{\begin{array}{ll}
a & (t \geq 0) \\
0 & (t<0)
\end{array},\right.
$$

where $h(t)$ is the Heaviside step function. The Fourier transformation of Eq. (A.1) is

$$
\begin{aligned}
\text { Noise }_{\mathrm{def}}(f) & =\int_{-\infty}^{\infty} \text { noise }_{\mathrm{def}}(t) e^{-j 2 \pi f t} d t \\
& =a \int_{0}^{\infty} e^{-j 2 \pi f t} d t \\
& =\frac{a}{2}\left\{\delta(f)+\frac{1}{j \pi f}\right\} .
\end{aligned}
$$

The noise level of the phase bias difference at frequency $f$ is given as the sum of the positive and negative frequency components of the noise spectrum Noise $_{\text {def }}(f)$. Therefore

$$
\begin{aligned}
\text { Noise }_{\text {spectrum }}(|f|) & =\mid \text { Noise }_{\text {spectrum }}(f)|+| \text { Noise }_{\text {spectrum }}(-f) \mid \\
& =a\left|\delta(f)+\frac{1}{j \pi f}\right|,
\end{aligned}
$$

is obtained.

In addition, this noise level is proportional to the number of changes of the selected port in a period of FFT. In other words, this noise level is inversely proportional to the shift interval of the selected port $T$.

On the basis of the above discussion, let us assume that a noise level is analyzed with $1 \mathrm{~Hz}$ frequency resolution. In this case, the period of FFT is one second, and the noise level induced by the phase bias difference is described as

$$
\text { Noise }_{\mathrm{PBD}}(f)=20 \log _{10}\left(\frac{a}{\pi f T}\right)-R S(f),
$$

where $R S(f)$ is the receiving sensitivity. 